\title{
Determination of Affinity Constants and Response Factors of the Noncovalent Dimer of Gramicidin by Electrospray Ionization Mass Spectrometry and Mathematical Modeling
}

\author{
Raghu K. Chitta, Don L. Rempel, and Michael L. Gross \\ Department of Chemistry, Washington University, Saint Louis, Missouri, USA
}

\begin{abstract}
The dimerization of gramicidin, a 15-residue membrane peptide, in solution can be viewed as a model for protein-protein interactions. We reported previously that the dimer can be observed when electrosprayed from organic solvents and that the abundances of the dimer depends on the dielectric constant of the solvent. Here, we report an effort to determine an affinity constant for the dimerization of gramicidin by using gas-phase abundance. Two issues affecting the determination are the electrospray-induced dissociation of the dimer and discrimination in the electrospray of the dimer compared with the monomer. Other methods developed for the purpose of determining affinity from mass spectral abundance do not address the dissociation of the complex in the gas phase or can not be applied for cases of low affinity constant, $K_{a}$. We present a mathematical model that uses the ratio of the signal intensities of the dimer and the monomer during a titration. The model also incorporates the dissociation and an electrospray ionization-response factor of the dimer for extracting the affinity constant for the dimerization of gramicidin. The dimerization constants from the new method agree within a factor of two with values reported in the literature. (J Am Soc Mass Spectrom 2005, 16, 1031-1038) (C 2005 American Society for Mass Spectrometry
\end{abstract}

S oft-ionization techniques now permit the introduction of biomolecule complexes into the gas phase [1-7]. Of interest to us is the dimer of gramicidin, which can enter the gas phase by electrospray ionization (ESI), and its abundance depends in a predictable way on the hydrophobicity of the organic solvent used in ESI [8]. The ability of gramicidin, a 15-residue membrane peptide, to form dimers in solution and in cell membranes determines its biological function. Its small size and stable structure have made it suitable for study by two-dimensional and threedimensional nuclear magnetic resonance, $\mathrm{X}$-ray crystallography and Circular Dichroism (CD) (for reviews, see [9-13]). Thus, its properties in the gas phase and the relation of those properties to those in solution is of importance.

Our specific goal is to determine the solution equilibrium constant for the dimer from the signal intensities for the monomer and the dimer in the gas phase. We considered literature methods whereby two signals

Published online May 23, 2005

Address reprint requests to Professor Michael L. Gross, Washington University, Department of Chemistry, Box 1134, Saint Louis, MO 63130, USA. E-mail: mgross@wustl.edu are measured by ESI mass spectrometry to obtain an affinity constant; these approaches make use of the measured intensity ratios of the complex and the protein in the gas phase when the ligand is in excess [14-18]. Although ESI can introduce noncovalent complexes of peptides and proteins to the gas phase [3, 19, 20], the differences in the ionization efficiency of proteins and protein-ligand complexes [21, 22] challenge the assumption that both are ionized with equal efficiency. The discrimination may not be serious for proteins and their complexes that have similar masses [23], explaining in part why direct measurements can be valid. For weakly bound complexes and for those that have significantly different masses than the peptide or protein (e.g., peptide-peptide and protein-protein complexes), problems with this approach will be more likely.

Dealing with these problems requires an understanding of the factors that affect ESI response. A partition model or another that estimates ESI response from charge gradients within the charged droplets was brought forward for that purpose [24-27]. Furthermore, a relative ESI response may be predictable from gasphase proton affinities, nonpolar properties, or chromatographic retention times of the analytes [28-30]. Success in these predictions also reveals aspects of the 
mechanism for ion formation in ESI and for discrimination during the process. For example, hydrophobic molecules that rise to the surface of an ESI-produced droplet have a better chance of being ionized. These ideas, however, are difficult to apply to a complex that is being studied for the first time.

Two related approaches can afford affinities: stability of unpurified proteins from rates of hydrogen/deuterium (H/D) exchange (SUPREX) [31] and proteinligand interactions by mass spectrometry, titration, and H/D exchange (PLIMSTEX) [32] (for a comparison, see [33]). These indirect approaches give equilibrium constants and avoid the problem of discrimination by using a one-signal $(\mathrm{m} / \mathrm{z})$ approach. The needed measurement is the extent of H/D amide exchange in solution as a function of ligand concentration (PLIMSTEX) or denaturant concentration (SUPREX). The extents of exchange are fixed in the protein by quenching the exchange and measured by mass spectrometry. A significant advantage of PLIMSTEX and SUPREX compared with direct equilibrium measurement is that the experiments can be conducted in biologically relevant buffers as the sample can be desalted in a quenched state before injection into the mass spectrometer. A disadvantage is that they rely on H/D amide exchange, which does complicate their experimental implementation. Therefore, we considered other direct methods that make use of signal intensities for the gas-phase complex (and the protein) but use titration and modeling to obtain equilibrium constants and response factors from ESI of molecules involved in equilibrium [17, 34].

In the most advanced two-signal method developed thus far [35], the concentrations of the protein and the ligand are varied, while the ratio of the protein-ligand concentrations is kept constant at unity. The signal intensities corresponding to the complex and the ligand are then measured by the mass spectrometer. A model fits the titration data and obtains both $K_{a}$ and a response factor. Although the method is an important step forward, it applies only to 1:1 systems. We presented a preliminary report [36] of another two-signal method to obtain an affinity constant for the binding of RNA to drugs. A limitation of these related methods is that they do not take into account the ESI-induced dissociation of the complex, which is likely when the complex is weakly bound [37, 38]. We are particularly concerned about this problem for gramicidin dimers [8].

Dissociation of weakly bound complexes in ESI, however, was considered in a new, one-signal approach whereby the measured abundance of the complex as a function of ligand concentration is modeled to give both a response factor and the equilibrium constant [34]. Although the approach affords good reproducibility, in part by using automation, the use of absolute intensities in a mass spectrum can pose problems when wide applicability is sought. First, local variations in the spray or in the spectrometer will affect intensity and the $K_{a}$ determination [39]. Second, for complexes with a low $K_{a}$, the method is difficult to apply because the signal intensity for the complex will be weak unless concentrations are high. Third, as the concentration of complex is increased, as it must in a titration, the charge in the ESI-produced droplet may be insufficient to ionize the same fraction of molecules as at a lower concentration [39-42].

Thus, we sought another mass spectrometry approach for determining the dimerization affinity of gramicidin. We recognize the need for a two-signal method that overcomes limitations of the previously described direct methods. The method development is motivated because (1) gramicidin has a low $K_{a}$ [43], indicating that the titration must be done at high concentrations to admit appreciable amounts of dimer in the gas phase; (2) a fraction of the dimer does dissociate during ESI (as reported in [8]); and (3) a model should assume no prior knowledge of the various physicochemical properties of the analytes or solvents. The model building starts with the one we used for PLIMSTEX [44] $]^{\circ}$ and ${ }^{\circ}$ is $^{\circ}$ described $^{\circ}$ in $^{\circ}$ the $e^{\circ}$ next ${ }^{\circ}$ section of this article.

\section{Experimental Procedures}

\section{Materials}

Gramicidin D was purchased from ICN Biochemicals (Costa Mesa, CA) and was used without further purification. The solvents ethanol and n-propanol were purchased from Sigma-Aldrich (Milwaukee, WI). The solutions of gramicidin were made by dissolving gramicidin in the appropriate solvent (i.e., ethanol and $n$-propanol) to a concentration of $2 \mathrm{mM}$. The solutions were then diluted to the requisite concentration before injection into the mass spectrometer.

\section{Mass Spectrometry}

Mass spectra of gramicidin in various solvents were acquired with the Q-Tof-Ultima (Micromass, Manchester, UK) operated in the positive-ion mode. This instrument was a tandem mass spectrometer, consisting of a quadrupole $(\mathrm{Q})$ mass analyzer, a quadrupole collision cell, and a second-stage time-of-flight (TOF) analyzer. Gramicidin was incubated in the ESI solvent before the mass spectrometry experiment. The ESI conditions were optimized for the highest sensitivity detection of the dimer. The needle voltage was $3 \mathrm{kV}$, and the cone voltage was $90 \mathrm{~V}$. The temperatures of the source block and for desolvation were $90{ }^{\circ} \mathrm{C}$. A flow rate of 10 $\mu \mathrm{L} / \mathrm{min}$ was used. All parameters (e.g., aperture to the TOF, transport voltage) were optimized to achieve maximum sensitivity and a mass resolving power of 10,000 (full width at half maximum).

ESI was performed by using $n$-propanol or ethanol solutions of gramicidin after saturating the solvents with $\mathrm{NaCl}$, by adding $0.1 \mathrm{mg}$ of the salt to the solvent, stirring with a vortex mixer, and centrifuging before introducing the supernatant into the mass spectrometer. 
The percent of dimer found in these experiments was calculated by integrating over the whole spectrum by using the integrate function of the "mass lynx" software of the spectrometer and then computing the ratio of the intensity of the ion signal of interest to that of the sum

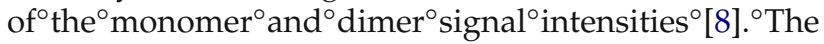
spectra were a sum of 2-s scans over $2 \mathrm{~min}$, and the spectra were smoothed twice using a three-point Savitzky-Golay method.

\section{Modeling}

To obtain affinity constants from the titration data, a model for the specific case of self-association was developed and implemented in Mathcad 2001i (MathSoft, Inc., Cambridge, MA). The model is a modified version

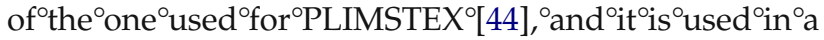
parameter-estimation mode that minimizes the difference between the experimental data representing the titration curve and the corresponding model outcome.

The unknown model parameters include the $\beta^{\prime}$ s involved in the self-association or self-binding of the monomer to form up to $N$ oligomers. Each $\beta_{j}$ value ( $j=$ $0-N)$ is the product of macroscopic binding constants $K$, $(i=1-j)$, as shown in eq 1 for the present case of gramicidin.

$$
\begin{aligned}
& \beta_{0}=1 \\
& \beta_{1}=K_{1} \\
& \beta_{2}=K_{1} K_{2}
\end{aligned}
$$

The equations for the $\beta^{\prime}$ s in terms of the K's, in general, depend on the reaction diagram. The $j$ th oligomer solution concentration is given by $\beta_{j}[\mathrm{Lig}]^{j+1}$ where $[\mathrm{Lig}]$ is the free solution monomer concentration. Using $\beta^{\prime} \mathrm{s}$ for defining the oligomer concentrations was more economical in terms of the equation expression and model implementation and subsumes the more general case when the reaction diagram is not sequential. Equations for the K's were obtained by solving the system shown in eq 1 and were used to calculate the affinity constants. The total analytical monomer concentration $[\mathrm{Lig}]_{\mathrm{T}}$, which is adjusted to bring the equilibrium to a given point on the titration curve, is accounted for in eq 2 because the $j$ th oligomer consumes $j+1$ monomer molecules.

$$
[L i g]_{\mathrm{T}}=\sum_{j=0}^{N}(j+1) \beta_{j}[L i g]^{j+1}
$$

The titration curve was produced by varying the independent variable $[\mathrm{Lig}]_{\mathrm{T}}$, causing a change in the free monomer solution concentration [Lig]. The monomer concentration $[\mathrm{Lig}]$, as a function of $[\mathrm{Lig}]_{\mathrm{T}}$ over the course of the titration, was obtained by numerically integrating (using the Mathcad "Rkadapt") the function $d[L i g] / d[L i g]_{\mathrm{T}}$, which is shown in eq 3 and was calculated as a reciprocal of $d[L i g]_{\mathrm{T}} / d[L i g]$ as derived from eq
2. The initial condition for the integration was that [Lig] $=0$, when $[\text { Lig }]_{\mathrm{T}}=0$.

$$
\frac{d[\mathrm{Lig}]}{d[\mathrm{Lig}]_{\mathrm{T}}}=\frac{1}{1+\sum_{i=1}^{N}(i+1)^{2} \beta_{i}[\mathrm{Lig}]^{i}}
$$

Model parameters also include the ESI-response factors $G_{i}$. The ESI-response factors describes the effect of ESI on the detection of the solution concentration of the oligomer complex, incorporating the discrimination of ESI relative to that of the monomer. ESI-response factor replaced the individual $\Delta D^{\prime}$ s that were used to describe amide exchange for PLIMSTEX. The function for the total relative intensity of an oligomer signal $S_{j}$ (in the gas phase) is shown in eq. 4 .

$$
S_{j}=f([L i g], \beta, j, G)=G_{j} \beta_{j}[L i g] \frac{1}{\beta_{0}+\sum_{i=1}^{N} G_{i} \beta_{i}[L i g]}
$$

where $G_{j}$ is the relative ESI-response factor for the $(j+$ 1)th oligomer compared with that of the monomer (e.g., the ESI-response factor for the dimer is $G_{1}$ ). Each product in eq 4 is a $G$-weighted solution concentration of an oligomer before a common factor of $[\mathrm{Lig}]$ was canceled from both the denominator and the numerator. The denominator corresponds to the sum of the signals for the monomer and all of the oligomers measured by the mass spectrometer.

The absolute total signal (the sum of peak intensities for the monomer and all the oligomers) was also compared with that predicted by the model, $S_{\mathrm{T}}$. The equation is (denominator in eq 4 before [ $\mathrm{Lig}]$ is canceled)

$$
S_{\mathrm{T}}=\beta_{0}[L i g]+\sum_{i=1}^{N} G_{i} \beta_{i}[L i g]^{i+1}
$$

This comparison was not used in the calculation of $K_{a}$, but it was used to check the appropriateness of the start values.

To allow the program to find a reliable solution for the unknown model parameters, the search was started at "good-guess" values for the parameters. Good-guess values were obtained by manually revising the guessed parameters until the computed model outcome compares favorably with the experimental data. The graphs for this comparison were also displayed by Mathcad. The Mathcad "Minimize" function was then used to refine the sought-after minima by minimizing the square root of the mean of the squares of the differences between the data and the corresponding model outcome over the relevant concentration range of the titration. The minimization was a nonlinear, quasiNewtonian method. All of the requirements for the correct application of non-linear least squares (NLLS) regression ${ }^{\circ}[45-47]^{\circ}$ are $^{\circ}$ assumed ${ }^{\circ}$ to ${ }^{\circ}$ be $^{\circ}$ satisfied. 
The search was implemented at two levels. Each level (one for the ESI-response factors and the other for the $\beta^{\prime}$ s) implements its own search process with a distinct set of parameters to be searched and a different root mean squares (RMS) method for comparison of the model outcome to the corresponding experiment data. For the highest level search, the parameters were the $\beta^{\prime}$ s, and the RMS was computed for the residuals between the total relative intensity of an oligomer signal $S_{j}$ (as in eq 4) and the corresponding experimental values for all points on the titration curve. For the next level search, the parameters were the ESI-response factors, and the RMS was computed for the residuals between the absolute total signal (as in eq 5) and the corresponding experimental values for the points at ligand concentrations that are lower than those that exhibit evidence of ESI signal saturation. This comparison was made after normalizing each curve to one at the highest used ligand concentration. Each trial in the search for the highest level causes a new search at the lower level because the trial parameter values at the highest level can affect the lower level search result (as is evident from the presence of $\beta$ in eq 5).

\section{Results and Discussion}

There are a variety of mass spectrometry-based methods to measure the affinity constant for self-association of gramicidin, which is the principal goal of this work. Most of the newer methods do deal with one or more of the problems of determining the equilibrium concentrations in solution by measuring corresponding ion abundances in the gas phase, as was described in the introduction. Our strategy is to use the modeling features of PLIMSTEX, without the need for H/D exchange, to determine the self-association of gramicidin. $\mathrm{We}^{\circ}$ modified $^{\circ}$ the $^{\circ}$ equations ${ }^{\circ}$ in $^{\circ}[44]^{\circ}$ for $^{\circ}$ application ${ }^{\circ}$ to self-association (in which the protein and ligand are the same) and used "self-titration" data to determine the affinity constants and ESI-response factors for the monomer and dimer.

\section{Application to Self-Association of Gramicidin}

We chose $n$-propanol and ethanol as solvents for the self-titration because they allow an appreciable amount of gramicidin dimer to be introduced into the gas phase [8]. ${ }^{\circ}$ Furthermore, ${ }^{\circ}$ the ${ }^{\circ}$ association ${ }^{\circ}$ constants ${ }^{\circ}$ for ${ }^{\circ}$ gramicidin in ethanol and $n$-propanol are available for comparison, having been determined by high-performance size exclusion chromatography (HPSEC) and fluorescence [43]..$^{\circ}{ }^{\circ}$ plot $^{\circ}$ of $^{\circ}$ relative ${ }^{\circ}$ amounts ${ }^{\circ}$ of $^{\circ}$ dimer $^{\circ}$ and ${ }^{\circ}$ monomer produced by ESI from $n$-propanol in the gas phase as a function ${ }^{\circ}$ of $^{\circ}$ analytical ${ }^{\circ}$ concentration $^{\circ}$ (points ${ }^{\circ}$ in $^{\circ}$ Figure $^{\circ} 1$ ) shows that the amount of dimer increases initially, with a corresponding decrease in the amount of the monomer, and then levels off at approximately $70 \%$, instead of asymptotically reaching $100 \%$. This is likely caused by dissociation of the dimer on ESI and is consistent

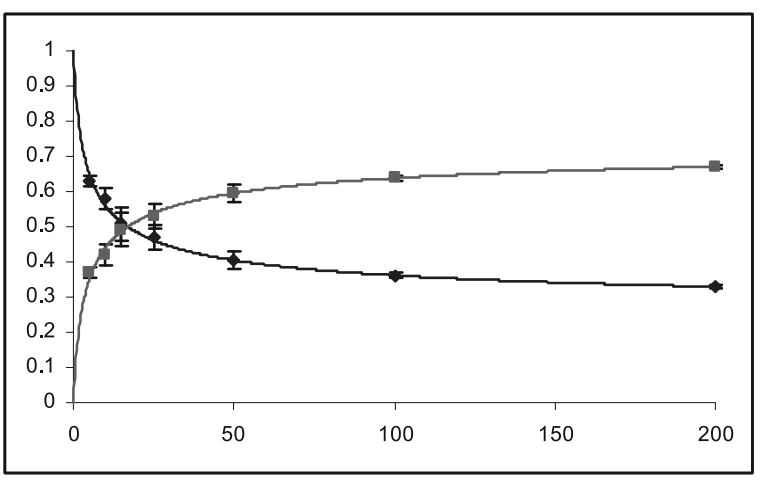

Figure 1. Variation of the amount of dimer (squares) and monomer (diamonds) of gramicidin in $n$-propanol as a function of analytical concentration. The squares and diamonds are the experimental data, and the solid curves are the theoretical (Th) fit to the data.

with the conclusions from our previous study where the signal intensity for dimer of gramicidin reached a maximum level of $68 \%$ as the dielectric constant of mixtures ${ }^{\circ}$ of $^{\circ}$ spray $^{\circ}$ solven $^{\circ}$ was $^{\circ}$ varied $^{\circ}[8]$.

The first issue we addressed when applying the model to gramicidin dimerization was the dissociation of $^{\circ}$ the ${ }^{\circ}$ dimer $^{\circ}$ accompanying $^{\circ} \mathrm{ESI}^{\circ}[8] .^{\circ} \mathrm{To}^{\circ}$ incorporate dissociation into the model, we assumed that a doubly charged dimer dissociates into two singly charged monomers, both of which would be detected. This assumption is reasonable because protein dimers in high charge states dissociate on collision-activated dissociation ${ }^{\circ}$ in $^{\circ} \mathrm{a}^{\circ}$ symmetric $^{\circ}$ fashion $^{\circ}[48],{ }^{\circ}$ and ${ }^{\circ}$ the ${ }^{\circ} 2+{ }^{\circ}$ state of the gramicidin dimer is a high charge state for that species ${ }^{\circ}[8] .{ }^{\circ} \mathrm{To}^{\circ}$ account $^{\circ}$ for $^{\circ}$ the ${ }^{\circ}$ dissociation ${ }^{\circ}$ in ${ }^{\circ}$ the ${ }^{\circ}$ modeling, the total relative intensity of the signals $S_{j}$ (eq 4 ) is rewritten for the gramicidin dimer and monomer as eqs $6 a$ and $6 b$, respectively:

$$
\begin{aligned}
S_{1}^{\prime} & =f([L i g], \beta, 1, d f, G) \\
& =\frac{(1-d f) G_{1}[D]}{[M]+2 d f G_{1}[D]+(1-d f) G_{1}[D]} \\
& =\frac{(1-d f) G_{1} \beta_{1}[L i g]}{\beta_{0}+2 d f G_{1} \beta_{1}[L i g]+(1-d f) G_{1} \beta_{1}[L i g]} \\
S_{0}^{\prime}= & f([L i g], \beta, 0, d f, G) \\
= & \frac{\beta_{0}+2 d f G_{1} \beta_{1}[L i g]}{\beta_{0}+2 d f G_{1} \beta_{1}[L i g]+(1-d f) G_{1} \beta_{1}[L i g]}
\end{aligned}
$$

where $d f$ is the fraction of the dimer $2+$ decomposing to two monomer ions in the process of electrospray. In the second line of eq $6 \mathrm{a},[D]$ and $[M]$ represent the solution concentrations of dimer and monomer, respectively. Finally, the expression for the absolute total signal was written to give eq 7:

$$
S_{T}^{\prime}=\beta_{0} L i g+2 d f G_{1} \beta_{1} L i g^{2}+(1-d f) G_{1} \beta_{1} L i g^{2}
$$

To solve for the parameters, the search described in the methods section was modified by adding a 
third level below the levels that solve for $\beta$ and $G$. The parameter searched at this new level is the dissociation factor, $d f$. The dissociation factor is best expressed toward the end of the titration curve (i.e., in the high concentration points where the curve is near its asymptote). This can be seen by noticing that eq $6 a$ becomes $1-d f / 1+d f$ in the limit of high total ligand concentration (i.e., when the $[D] \gg[M]$ ). Accordingly, the RMS for this new search was computed from the residuals between the total relative intensity of the dimer signal $S_{j}$ (as in eq 6a) and the corresponding experimental values for the three high ligand concentration points on the titration curve. In the self-titration curve of gramicidin in propanol (Figure ${ }^{\circ} 1$ ), ${ }^{\circ}$ for ${ }^{\circ}$ example, ${ }^{\circ}$ the ${ }^{\circ}$ points ${ }^{\circ}$ for ${ }^{\circ}$ concentrations $\geq 50 \mu \mathrm{M}$ were used for the calculation of $d f$.

The $K_{a}$ values for the dimerization of gramicidin from the model agree well with the literature values in the two solvent systems, supporting the validity of the approach. The fitted model returned a value of $5 \pm 1$ for the ESI-response factor for the dimer when introduced by ESI from $n$-propanol and a $K_{a}=5 \pm 3 \times 10^{4}$ (literature value for $K_{a}$ is ${ }^{\circ} 1.4^{\circ} \times{ }^{\circ} 10^{4^{\circ}}$ [43]). ${ }^{\circ}$ The ${ }^{\circ}$ model ${ }^{\circ}$ fits the data with an RMS value of 0.2. For dimerization in ethanol, the model returned a value of $1.3 \pm 0.2$ for the ESI-response factor for the dimer and a value of $6 \pm 1 \times$ $10^{3}$ for $K_{a}$ (literature value for $K_{a}$ is ${ }^{\circ} 1^{\circ} \times{ }^{\circ} 10^{4^{\circ}}$ [43]). Reasons for the differences in ESI-response factors for gramicidin in $n$-propanol and in ethanol and the implications of response factor for mechanism of ESI are discussed later.

The calculated values of $d f$ in $n$-propanol and ethanol are $0.15 \pm 0.01$ and $0.19 \pm 0.01$, respectively. We reasoned ${ }^{\circ}$ in $^{\circ}$ our $^{\circ}$ earlier ${ }^{\circ}$ publication $[8]^{\circ}$ that ${ }^{\circ}$ dissociation of some dimer must occur on ESI because the dimer signal, which increases linearly as the dielectric constant ${ }^{\circ}$ of $^{\circ}$ the ${ }^{\circ} \mathrm{ESI}^{\circ}$ solven $t^{\circ}$ decreases, ${ }^{\circ}$ levels ${ }^{\circ}$ off ${ }^{\circ}$ at $^{\circ} 68 \%$ [8]. Using eq $6 \mathrm{a}$ in the limit for those data returns a value of 0.19 for $d f$, which agrees well with the values calculated here. Although the internal energy of ESI-produced ions $^{\circ} \operatorname{can}^{\circ}$ depend $^{\circ}$ on $^{\circ}$ solvent $^{\circ}$ composition $^{\circ}[49]^{\circ}$, this dependence occurred when one of the components (glycerol) of a binary mixture had considerably lower volatility than the other'(water) [49]. The ${ }^{\text {Thear }}{ }^{\circ}$ constancy of $d f$, as shown for the two solvents used here, suggests that between solvents of comparable vapor pressure, the internal energy of ESI-produced ions is similar.

One concern when determining affinity constants from direct intensity measurements is that solvent evaporation during ESI skews the results for the ESIresponse factor $G$ and $\beta_{1}$ because the analyte concentration in the droplet increases with solvent evaporation. We tested this computationally by increasing the concentration $[\mathrm{Lig}]_{\mathrm{T}}$ to values above the experimentalsolution concentrations and evaluated its effect on $G$ and $\beta_{1}$ by using eq 8 together with equations $6 \mathrm{a}$ and $6 \mathrm{~b}$. An equation for $[\mathrm{Lig}]_{\mathrm{T}}$ for the specific case of monomerdimer equilibrium becomes

$$
[L i g]=\frac{-1+\sqrt{1+8 \beta_{1}[L i g]_{T}}}{4 \beta_{1}}
$$

When a fit to the synthesized data is achieved, the resulting $\beta_{1}$ is higher, but the resulting $G$ remains nearly constant because it does not appear in the eq 8 , which is the only equation where $[\mathrm{Lig}]_{\mathrm{T}}$ appears explicitly. This outcome might be anticipated by noting that the $\beta_{1}-[L i g]$ product depends solely on the $\beta_{1}-[L i g]_{\mathrm{T}}$ product, as can be seen by multiplying both sides of eq 8 by $\beta_{1}$. Because the $K_{a}^{\prime}$ s determined by this mass spectrometric method are in reasonable agreement with literature values, we conclude that the concentrating effect of the ESI process does not significantly affect the affinity-constant determination. It may be that the ESI process occurs faster than the shift in the monomer-dimer equilibrium. We reported earlier that there were no concentrating effects for gramicidin when electrosprayed from organic solvents under ${ }^{\circ} \operatorname{similar}^{\circ}$ experimental $^{\circ}$ conditions $^{\circ}[8],{ }^{\circ}$ and ${ }^{\circ}$ Kebarle $^{\circ}$ and ${ }^{\circ}$ coworkers $^{\circ}[23]^{\circ}$ arrived ${ }^{\circ} a^{\circ}$ the ${ }^{\circ}$ same $^{\circ}$ conclusion ${ }^{\circ}$ for ${ }^{\circ}$ the nanospray of protein-ligand complexes from aqueous solutions.

We wish to compare the outcome of the method we report ${ }^{\circ}$ rere $^{\circ}$ with ${ }^{\circ}$ that ${ }^{\circ}$ of $^{\circ}$ the ${ }^{\circ}$ one-signal ${ }^{\circ}$ method $[34]^{\circ}$ that determines $K_{a}$ by relying solely on the absolute intensity of the complex as a function of added ligand amount. We implemented this one-signal method and found that it gave a $K_{a}$ that does not agree with the results calculated ${ }^{\circ}$ previously $^{\circ}$ or $^{\circ}$ reported $^{\circ}$ in $^{\circ}$ literature ${ }^{\circ}[43]^{\circ}$ (a comparison ${ }^{\circ}$ is $^{\circ}$ in $^{\circ}$ Table $\left.^{\circ} 1\right) .^{\circ}$ It $^{\circ}$ may $^{\circ}$ be $^{\circ}$ that $^{\circ}$ there $^{\circ}$ is insufficient charge on the ESI droplet to charge all the dimer molecules, and this would invalidate a simple relationship between the absolute intensity in the gas phase and the concentration in solution.

\section{Robustness of the Model}

That $\beta_{1}$ and $G$ appear together in the model signal functions (eqs 6a, 6b and 7), suggests that at first thought, we can discern only the $\beta_{1} G$ product from the experiment data. The signal functions are shown in terms of the free ligand concentration. The comparison between model and experimental data for the purpose of the fit, however, was made as a function of the

Table 1. Comparison of the association constant of gramicidin in $n$-propanol determined by using the model developed in this article, ${ }^{\circ}$ that ${ }^{\circ}$ from ${ }^{\circ}$ the ${ }^{\circ}$ model $^{\circ}$ of ${ }^{\circ}$ Tjernberg $\left.^{\circ} \mathrm{et}^{\circ}{ }^{\circ}{ }^{\circ}{ }^{\circ}[34]\right]^{\circ}$ and ${ }^{\circ}$ that reported ${ }^{\circ}{ }^{\circ}{ }^{\circ}$ literature ${ }^{\circ}[43]$

\begin{tabular}{lcc}
\hline Method & $K_{a}\left(\mathrm{M}^{-1}\right)$ & Response factor \\
\hline \hline $\begin{array}{l}\text { HPLC and fluorescence } \\
\text { spectroscopy [43] }\end{array}$ & $(1.5 \pm 0.2) 10^{4}$ & N/A \\
$\begin{array}{l}\text { Ratio of intensities } \\
\text { (this report) }\end{array}$ & $(5 \pm 3) 10^{4}$ & $5 \pm 1$ \\
$\begin{array}{l}\text { Absolute dimer } \\
\text { intensity [34] }\end{array}$ & $(1.1 \pm 0.3) 10^{9}$ & $(2.9 \pm 0.5) 10^{3}$ \\
\hline
\end{tabular}

HPLC, high-performance liquid chromatography; N/A, not applicable. 
solution total ligand concentration. The consequence of this, for the current case of $N=1$, can be visualized by substituting the free ligand concentration $[\mathrm{Lig}]$ into the expression given in eq 7. The formal paring of $\beta_{1}$ and $G$ as a product is disrupted. The method of analysis is further assisted by the intermediate search level, which compares the normalized ${ }^{\circ}$ version ${ }^{\circ}$ of the ${ }^{\circ}$ model ${ }^{\circ}$ absolute total signal (eq 7) with corresponding experimental result. The shape of the absolute total signal is strongly influenced by $G$ for moderate values and is strictly convex if $((1+d f) G-2)>0$, strictly concave if $((1+$ $d f) G-2)<0$, and linear, otherwise. This can be seen from the expression of the second derivative of the absolute signal as shown in eq 9 .

$$
\begin{aligned}
\frac{\partial^{2} S_{T}^{\prime}}{\partial[L i g]_{T}^{2}} & =\frac{\partial}{\partial[L i g]}\left\{\frac{\partial S_{T}^{\prime}}{\partial[L i g]_{T}}\right\} \frac{\partial[L i g]}{\partial[L i g]_{T}} \\
& =\frac{2\left[(1+d f) G_{1}-2\right] \beta_{1}}{\left(1+4 \beta_{1}[L i g]\right)^{3}}
\end{aligned}
$$

We tested the model to see if it is sufficiently well conditioned for the intended use by asking whether the model can simultaneously give both $K_{a}$ and $G$. If the number of variables is too large for the amount of experimental data or if $K_{a}$ and $G$ are interrelated, we would expect the fit answers to be sensitive to start values. Within experimental error, a search converged to the same values (data not shown) when the start values were varied by 10,20 , and $50 \%$, supporting the conclusion that there is a well-defined minimum for the parameters.

We also tested the model by evaluating its sensitivity to the concentration of gramicidin. To perform the robustness tests, we chose the model parameters $K_{a}$ and $G$ to be $3 \times 10^{5}$ and 6, respectively, and a $d f$ of 0.16 and synthesized the intensity ratios for various analytical concentrations to obtain a "synthetic" titration curve. The analytical concentrations were then perturbed by increasing and decreasing the values by 5,25 , and $50 \%$ of the values used in the synthesis. The resulting tables of concentrations and intensity ratios were fit to obtain new ${ }^{\circ}$ affinity ${ }^{\circ}$ Constants $^{\circ}$ (results ${ }^{\circ}$ in Table 2)..$^{\circ}$ Although the $K_{a}$ values vary, they differ only by a small extent, similar to the variations in concentration even when

Table 2. Test of the robustness of the model with respect to perturbations of the gramicidin concentrations

\begin{tabular}{lcc}
\hline Concentration perturbed by & $K_{a}^{\text {start }} / 10^{5}$ & $K_{a}^{\text {srch result } / 10^{5}}$ \\
\hline \hline- & 3.7 & 3.7 \\
$\downarrow$ by $5 \%$ & 3.7 & 3.3 \\
$\uparrow$ by $5 \%$ & 3.7 & 3.0 \\
$\downarrow$ by $25 \%$ & 3.7 & 4.1 \\
$\uparrow$ by $25 \%$ & 3.7 & 2.5 \\
$\downarrow$ by $50 \%$ & 3.7 & 0.6 \\
$\uparrow$ by $50 \%$ & 3.7 & 0.2 \\
\hline
\end{tabular}

All the trials returned a value of 5.7 for the ESI-response factor and 0.16 for the dissociation factor. concentrations had been perturbed by $50 \%$. The model is, thus, quite robust with respect to errors in concentration. ${ }^{\circ}$ Gabelica ${ }^{\circ}$ et $^{\circ}$ al. ${ }^{\circ}[35],{ }^{\circ}$ in $^{\circ}$ their $^{\circ}$ development ${ }^{\circ}$ of $^{\circ}$ a mass spectrometric method for affinities, found that their method requires accurate equimolar mixtures of the reactants be varied to achieve changes in mass spectrometric signals that they relate to variations in solution. They reported that when the concentrations are not equimolar or vary by $10 \%$, their model returns $K_{a}$ values that can change by two orders of magnitude.

To test whether the model would be robust if only a fraction of the dissociated dimer was detected in the monomer signal, we synthesized data with the set of parameters used for the aforementioned concentration tests, but now assumed that only a fraction of the dissociated dimer is incorporated into the monomer signal and the rest is not detected. The synthesis was done, for example, where only one-third of the dissociated dimer was detected by replacing $2 d f$ in eqs $6 a$ and $6 \mathrm{~b}$ with $2 d f / 3$. When the search converged for these data sets, the model compensated for this by decreasing the $d f$ by approximately the same fraction and keeping $K_{a}$ and $G$ constant. This shows that the assumption that all dimer must dissociate into detectable monomers is not crucial for the calculation of $K$ and $G$ but does affect the determination of $d f$.

\section{ESI-Response Factor and Implication for ESI}

Is there a physical meaning to the ESI-response factor and why is the ESI-response factor four times larger for the dimer when sprayed from $n$-propanol than from ethanol? These are two important questions. A response factor that is greater than 1 indicates that the dimer ionizes better by ESI and is expressed better in the gas phase than is the monomer. The sensitivity to solvent, however, is much larger than the variation of the dielectric constant or the surface tension of the two solvents. Although n-propanol has a lower dielectric constant and higher surface tension than ethanol, these properties are different from those of ethanol by factors of 1.2 and 1.1, respectively. Although these factors may play a role in the differences, they can not play a major role.

For gramicidin, dimerization causes concealment of hydrophilic ${ }^{\circ}$ centers ${ }^{\circ}\left[9^{\circ}-11\right] .{ }^{\circ}$ Thus, $^{\circ}$ the ${ }^{\circ}$ dimer, $^{\circ}$ which ${ }^{\circ}$ is more hydrophobic than the monomer, should rise more readily ${ }^{\circ}$ to $^{\circ}$ the ${ }^{\circ}$ surface ${ }^{\circ}$ of $^{\circ}$ ESI-produced ${ }^{\circ}$ droplets $^{\circ}[28,29]$ and be ionized with more facility, causing the ESIresponse factor $G$ to be greater than 1 for both ethanol and $n$-propanol. To explain the difference between the response factors in $n$-propanol and ethanol, we suggest that because $n$-propanol has a higher boiling point than ethanol, it evaporates more slowly in the ESI process. Its droplets live longer, providing more time for the dimer to concentrate on the surface and produce a higher ESI-response factor. 


\section{Conclusion}

The affinity constants for self-association of gramicidin in two solvents (ethanol and n-propanol), as determined by the mass spectrometric approach outlined in this article, agree within a factor of two with those determined by HPSEC and fluorescence. This approach may be useful for determining affinity constants for other self-associations, including those of proteins, when an assumption can not be made about the sensitivity factor. The changes required for adaptation to protein-ligand or protein-protein binding are minimal and become obvious when the equations in this article are compared with those made in the development of PLIMSTEX ${ }^{\circ}[44]^{\circ}{ }^{\circ}$ In ${ }^{\circ}$ dealing ${ }^{\circ}$ with $^{\circ}$ protein-ligand interactions, two degrees of freedom can be achieved in the procedure by keeping the protein concentration constant and varying the ligand concentration or by keeping the ligand concentration constant and varying that of the protein, similar to an approach we described elsewhere ${ }^{\circ}[36]^{\circ}{ }^{\circ}$ This $^{\circ}{ }^{\text {approach }}{ }^{\circ}$ may $^{\circ}$ give $^{\circ}$ better $^{\circ}$ values for the sensitivity factor and the equilibrium constant when a dissociation factor is incorporated into it.

Nevertheless, the approach, like all approaches that deduce affinity constants from mass spectrometric signals, has a drawback. We must assume that the ratio of the mass spectrometric intensities represent ratios of concentrations in solution even though mass spectrometric absolute intensities are not strictly proportional to solution concentrations (i.e., $S_{j} \neq k\left[M_{j+1}\right]$ ). This means that the detector shows no discrimination of the complex to the monomer, which may not be true for a multichannel plate (MCP) such as that used in the mass spectrometer employed here. Although this drawback exists, the approach developed for gramicidin, unlike the ${ }^{\circ}$ absolute ${ }^{\circ}$ intensity ${ }^{\circ}$ method $^{\circ}$ of $^{\circ}$ Tjernberg $^{\circ}$ et $^{\circ}$ al..$^{\circ}[34]$, should work over a wider range of circumstances. Furthermore, this approach is more tolerant to errors in concentration ${ }^{\circ}$ than $^{\circ}$ the ${ }^{\circ}$ method $^{\circ}$ of $^{\circ} \mathrm{Gabelica}^{\circ} \mathrm{et}^{\circ}{ }^{\circ} \mathrm{l}^{\circ}[35]$.

This approach may also be useful for studying the ESI process. Systematic studies of equilibrium systems may give trends of $G$ and $d f$ values that may be useful in probing ionization mechanisms and seeking "ideal" ESI systems that give $G=1$ and $d f=0$.

\section{Acknowledgments}

The authors thank the National Center for Research Resources of the National Institutes of Health for financial support (grant 2P41RR00954).

\section{References}

1. Strupat, K.; Metelmann, W. Mass Spectrometry of Peptides and Proteins---Principles and Features of Electrospray/Ionization-Mass Spectrometry (ESI-MS) and Matrix-Assisted Laser Desorption/Ionization-Mass Spectrometry. Supramolecular Structure and Function 7. Proceedings of the International Summer School on Biophysics, 7, Rovinj, Croatia, September 14-25, 2000, 2001; pp 27-44.
2. Ayed, A.; Krutchinsky, A. N.; Ens, W.; Standing, K. G.; Duckworth, H. W. Quantitative Evaluation of Protein-Protein and Ligand-Protein Equilibria of a Large Allosteric Enzyme by Electrospray Ionization Time-Of-Flight Mass Spectrometry. Rapid Commun. Mass Spectrom. 1998, 12, 339-344.

3. Ganem, B.; Henion, J. D. Going Gently into Flight: Analyzing Noncovalent Interactions by Mass Spectrometry. Bioorg. Med. Chem. 2003, 11, 311-314.

4. Light-Wahl, K. J.; Schwartz, B. L.; Smith, R. D. Observation of the Noncovalent Quaternary Associations of Proteins by Electrospray Ionization Mass Spectrometry. J Am Chem Soc 1994, 116, 5271-5278

5. Sobott, F.; Robinson, C. V. Protein Complexes Gain Momentum. Curr. Opin. Struct. Biol. 2002, 12, 729-734.

6. Robinson, C. V. Characterization of Multiprotein Complexes by Mass Spectrometry. Protein-Protein Interact. 2002, 227-240.

7. Miranker, A. D. Protein Complexes and Analysis of Their Assembly by Mass Spectrometry. Curr. Opin. Struct. Biol. 2000, 10, 601-606

8. Chitta, R. K.; Gross, M. L. Electrospray Ionization-Mass Spectrometry and Tandem Mass Spectrometry Reveal Self-Association and Metal-Ion Binding of Hydrophobic Peptides: A Study of the Gramicidin Dimer. Biophys. J. 2004, 86, 473-479.

9. Wallace, B. A. Gramicidin, a "Simple" Ion Channel. Curr. Top. Membr Transport 1988, 33, 35-50.

10. Wallace, B. A. Recent Advances in the High Resolution Structures of Bacterial Channels: Gramicidin A. J. Struct. Biol. 1998, 121, 123-141.

11. Wallace, B. A. Gramicidin Channels and Pores. Ann. Rev. Biophys. Biophys. Chem. 1990, 19, 127-157.

12. Veatch, W. R.; Fossel, E. T.; Blout, E. R. The Conformation of GRAMIcidin A. Biochemistry 1974, 13, 5249-5256.

13. Urry, D. W. The Gramicidin A Transmembrane Channel: A Proposed pi(L,D) Helix. Proc. Natl. Acad. Sci. U.S.A. 1971, 68, 672-676.

14. Sannes-Lowery, K. A.; Griffey, R. H.; Hofstadler, S. A. Measuring Dissociation Constants of RNA and Aminoglycoside Antibiotics by Electrospray Ionization Mass Spectrometry. Anal. Biochem. 2000, 280, 264-271.

15. Leary, J. A. Noncovalent Enzyme-Ligand Complexes: Gas and Solution Phase Studies. Abstracts of Papers, 227th ACS National Meeting; Anaheim, CA, March 28-April 1, 2004; paper PHYS176.

16. Bligh, S. W. A.; Haley, T.; Lowe, P. N. Measurement of Dissociation Constants of Inhibitors Binding to Src SH2 Domain Protein by Non-Covalent Electrospray Ionization Mass Spectrometry. J. Mol. Recognit. 2003, 16, 139-147.

17. Rosu, F.; Gabelica, V.; Houssier, C.; De Pauw, E. Determination of Binding Constants of Oligonucleotide Complexes with Minor Groove Binders by Electrospray Ionization Mass Spectrometry. Comparison with Fluorescence Titration Data. Adv. Mass Spectrom. 2001, 15, 795-796.

18. Zhang, S.; Van Pelt, C. K.; Wilson, D. B. Quantitative Determination of Noncovalent Binding Interactions Using Automated Nanoelectrospray Mass Spectrometry. Anal. Chem. 2003, 75, 3010-3018.

19. Fenselau, C. Analysis of Metalloproteins by Electrospray Mass Spectrometry. Adv. Mass Spectrom. 2001, 15, 151-166.

20. Veenstra, T. D. Electrospray Ionization Mass Spectrometry in the Study of Biomolecular Non-Covalent Interactions. Biophys. Chem. 1999, 79, 63-79.

21. Sojo, L. E.; Lum, G.; Chee, P. Internal Standard Signal Suppression by Co-Eluting Analyte in Isotope Dilution LC-ESIMS. Analyst (Cambridge, United Kingdom) 2003, 128, 51-54.

22. Liang, H. R.; Foltz, R. L.; Meng, M.; Bennett, P. Ionization Enhancement in Atmospheric Pressure Chemical Ionization and Suppression in Electrospray Ionization between Target 
Drugs and Stable-Isotope-Labeled Internal Standards in Quantitative Liquid Chromatography/Tandem Mass Spectrometry. Rapid Commun. Mass Spectrom. 2003, 17, 2815-2821.

23. Peschke, M.; Verkerk, U. H.; Kebarle, P. Features of the ESI Mechanism That Affect the Observation of Multiply Charged Noncovalent Protein Complexes and the Determination of the Association Constant by the Titration Method. J. Am. Soc. Mass Spectrom. 2004, 15, 1424-1434.

24. Enke, C. A Predictive Model for Matrix and Analyte Effects in Electrospray Ionization of Singly-Charged Ionic Analytes. Anal. Chem. 1997, 69, 4885-4893.

25. Sherman, C. L.; Brodbelt, J. S. An Equilibrium Partitioning Model for Predicting Response to Host-Guest Complexation in Electrospray Ionization Mass Spectrometry. Anal. Chem. 2003, 75, 1828-1836.

26. Zhou, S.; Cook, K. D. A Mechanistic Study of Electrospray Mass Spectrometry: Charge Gradients within Electrospray Droplets and Their Influence on Ion Response. J. Am. Soc. Mass Spectrom. 2001, 12, 206-214.

27. Zhou, S. In Univ. of Tennessee, Knoxville, TN, USA. FIELD URL:, 2000, p 207.

28. Cech, N. B.; Enke, C. G. Relating Electrospray Ionization Response to Nonpolar Character of Small Peptides. Anal. Chem. 2000, 72, 2717-2723.

29. Cech, N. B.; Enke, C. G. Effect of Affinity for Droplet Surfaces on the Fraction of Analyte Molecules Charged during Electrospray Droplet Fission. Anal. Chem. 2001, 73, 4632-4639.

30. Cech, N. B.; Krone, J. R.; Enke, C. G. Predicting Electrospray Response from Chromatographic Retention Time. Anal. Chem. 2001, 73, 208-213.

31. Powell, K. D.; Ghaemmaghami, S.; Wang, M. Z.; Ma, L.; Oas, T. G.; Fitzgerald, M. C. A General Mass Spectrometry-Based Assay for the Quantitation of Protein-Ligand Binding Interactions in Solution. J. Am. Chem. Soc. 2002, 124, 10256-10257.

32. Zhu, M. M.; Rempel, D. L.; Du, Z.; Gross, M. L. Quantification of Protein-Ligand Interactions by Mass Spectrometry, Titration, and H/D Exchange: PLIMSTEX. J. Am. Chem. Soc. 2003, 125, 5252-5253.

33. Breuker, K. New Mass Spectrometric Methods for the Quantification of Protein-Ligand Binding in Solution. Angew. Chem. Int. Ed. 2004, 43, 22-25.

34. Tjernberg, A.; Carnoe, S.; Oliv, F.; Benkestock, K.; Edlund, P.-O.; Griffiths, W. J.; Hallen, D. Determination of Dissociation Constants for Protein-Ligand Complexes by Electrospray Ionization Mass Spectrometry. Anal. Chem. 2004, 76, 4325-4331.

35. Gabelica, V.; Galic, N.; Rosu, F.; Houssier, C.; De Pauw, E. Influence of Response Factors on Determining Equilibrium Association Constants of Non-Covalent Complexes by Elec- trospray Ionization Mass Spectrometry. J. Mass Spectrom. 2003, 38, 491-501.

36. Gross, J.; Xavier, A; Gross, M. RNA Neomycin Complexes Analyzed by Electrospray and UV-MALDI-MS. Abstracts of Papers, 49th ASMS Conference on Mass Spectrometry and Allied Topics; Chicago, IL; June 2001.

37. Wu, Q.; Gao, J.; Joseph-McCarthy, D.; Sigal, G. B.; Bruce, J. E.; Whitesides, G. M.; Smith, R. D. Carbonic Anhydrase-Inhibitor Binding: From Solution to the Gas Phase. J. Am. Chem. Soc. 1997, 119, 1157-1158.

38. Robinson, C. V.; Chung, E. W.; Kragelund, B. B.; Knudsen, J.; Aplin, R. T.; Poulsen, F. M.; Dobson, C. M. Probing the Nature of Noncovalent Interactions by Mass Spectrometry. A Study of Protein-CoA Ligand Binding and Assembly. J. Am. Chem. Soc. 1996, 118, 8646-8653.

39. Cech, N. B.; Enke, C. G. Practical Implications of Some Recent Studies in Electrospray Ionization Fundamentals. Mass Spectrom. Rev. 2002, 20, 362-387.

40. Tang, L.; Kebarle, P. Dependence of Ion Intensity in Electrospray Mass Spectrometry on the Concentration of the Analytes in the Electrosprayed Solution. Anal. Chem. 1993, 65, 36543668.

41. Zook, D. R.; Bruins, A. P. On Cluster Ions, Ion Transmission, and Linear Dynamic Range Limitations in Electrospray (Ionspray) Mass Spectrometry. Int. J. Mass Spectrom. Ion Process. 1997, 162, 129-147.

42. Ikonomou, M. G.; Blades, A. T.; Kebarle, P. Investigations of the Electrospray Interface for Liquid Chromatography/Mass Spectrometry. Anal. Chem. 1990, 62, 957-967.

43. Braco, L.; Bano, C.; Chillaron, F.; Abad, C. Dimer-Monomer Conformational Equilibrium of Gramicidin A in 1-Alkanols as Studied by H.P.L.C. and Fluorescence Spectroscopy. Int. J. Biol. Macromol. 1988, 10, 343-348.

44. Zhu, M. M.; Rempel, D. L.; Gross, M. L. Modeling Data from Titration, Amide H/D Exchange, and Mass Spectrometry to Obtain Protein-Ligand Binding Constants. J. Am. Soc. Mass Spectrom. 2004, 15, 388-397.

45. Johnson, M. L. Why, When, and How Biochemists Should Use Least Squares. Anal. Biochem. 1992, 206, 215-225.

46. Johnson, M. L. Outliers and Robust Parameter Estimation. Meth. Enzymol. 2000, 321, 417-424.

47. Johnson, M. L. Parameter Correlations While Curve Fitting. Meth. Enzymol. 2000, 321, 424-446.

48. Jurchen, J. C.; Williams, E. R. Origin of Asymmetric Charge Partitioning in the Dissociation of Gas-Phase Protein Homodimers. J. Am. Chem. Soc. 2003, 125, 2817-2826.

49. Collette, C. D. P. E. Calibration of the Internal Energy Distribution of the Ions Produced by Electrospray. Rapid Commun. Mass Spectrom. 1998, 12, 165-170. 